\title{
ETUDE DES ERREURS D'ESTIMATION DES POPULATIONS PAR LA METHODE DES CAPTURES SUCCESSIVES (DeLURY, 2 CAPTURES)
} ET DES CAPTURES-RECAPTURES (PETERSEN)

\author{
Max LAURENT \\ Institut National de la Recherche Agronomique \\ Centre de Recherches Hydrobiologiques \\ B.P. 79,64200 BIARRITZ
}

\begin{abstract}
RESUME
L'estimation des populations naturelles par capture-recapture et par captures successives est souvent entachée d'erreur car, dans de nombreux cas, l'hypothèse fondamentale d'égalité des probabilités de captures pour tous les individus dans le temps et dans l'espace n'est pas respectée. Dans le cas des populations de poissons envisagés ici. les captures ont lieu par la pêche élecstrique. On a pu chiffrer l'ordre de grandeur des erreurs systématiques faites sur l'estimation des peuplements, en fonction des conditions particulières, biotiques et abiotiques, des différents milieux inventoriés.
\end{abstract}




\section{INTRODUCTION}

L'estimation des populations est souvent entachée d'erreur, car l'hypothèse fondamentale d'égalité des probabilités de capture pour tous les individus, dans le temps et dans l'espace, n'est jamais respectée dans la réalité. Le but de ce travail est l'étude des erreurs sur les estimations entrainées par les différences de probabilité. Cette étude a une portée générale mais, pour fixer les idees, on considerera une population de poissons en étudiant d'abord le cas où l'efficacité varie d'une pêche à l'autre, puis celui où, au cours d'une même pêche, on se trouve devant deux "sous-populations" capturables avec des efficacités différentes. Chaque cas ètudié sera suivi d'un exemple numérique montrant l'importance de l'erreur.

\section{NOTATIONS UTILISEES DANS LE TEXTE}

$$
\mathrm{P}=\text { population réelle }
$$

$\Lambda$ $\mathrm{P}_{\mathrm{L}}=$ estimation par la méthode DeLURY
$\Lambda$
$\mathrm{P}_{\mathrm{P}}=$ estimation par la méthode PETERSEN
$\mathrm{C}_{1}=$ nombre de poissons capturés en 1ere pêche DeLURY
$\mathrm{C}_{2}=$ nombre de poissons capturés en $2^{\mathrm{e}}$ pêche DeLURY
$\mathrm{m}=\mathrm{C}_{1}=$ nombre de poissons capturés en 1 ere pêche PETERSEN
$r=$ nombre de poissons marqués capturés en $2^{e}$ pêche PETERSEN
$\mathrm{u}=\mathrm{C}_{2}=$ nombre de poissons capturés en $2^{\mathrm{e}}$ pêche PETERSEN

\section{EFFICACITE VARIABLE D'UNE PECHE A L'AUTRE}

$x=$ efficacité en 1 ere pêche (PETERSEN et DeLURY) et sur les marqués (PETERSEN) en $2^{e}$ pèche

$y=$ efficacité en $2^{e}$ pêche (DeLURY) et sur les non marqués en $2^{e}$ pêche (PETERSEN).

Dans le cas de la méthode PETERSEN, lorsque l'efficacité sur les marqués en $2^{e}$ pêche est différente de l'efficacité de la première pêche, la complexité des calculs empêche de tirer des conclusions claires.

\subsection{Méthode DeLURY}

$\mathrm{C}_{1}=\mathrm{Px}$

$C_{2}=(P-P x) y=P(1-x) y$

$$
{\stackrel{\Lambda}{P_{L}}}=\frac{C^{2}{ }_{1}}{C_{1}-C_{2}}=\frac{P^{2} x^{2}}{P x-P(1-x) y}=P \frac{x^{2}}{x-y+x y}
$$


$1 \quad \mathrm{~N}$

PL s'écrit $P .-\frac{1}{D}$, avec $N=x^{2}$ et $D=x-y+x y$, et pour connaitre le sens de l'erreur (surestimation ou sous-estimation), on peut étudier le signe de $N-D$ :

$N-D=x^{2}-x+y-x y$

$=(1-x)(y-x)$.

On voit que $N-D$ est du signe de $y-x, x$ étant toujours inférieur à 1.

1

Donc, si $y>x, N-D>O$, et $P_{L}>P$ : surestimation

$\Lambda$

si $y<x, N-D<O$, et $P_{L}<P$ : sous-estimation.

\section{Exemple numérique}

Soit $P=200$ et une efficacité en 1ere pêche $x=0,6$ ou $60 \%$ et en 2e pêche $y=0,4$ ou $40 \%$.

$\Lambda \quad .1$ 0,36

L'estimation $P_{\mathrm{L}}$ sera : $\mathrm{PL}_{\mathrm{L}}=200 \times \overline{0.2+0.24}$

$\Lambda=200 \times 0.82=164$ soit une sous-estimation de $18 \%$. $\mathrm{Si}$, avec la même population $\mathbf{P}=200$, on suppose $\mathrm{x}=0,4$ et $\mathrm{y}=0,6$, on obtient :

$\hat{P}_{L_{2}}=200 \times \frac{0.16}{0,24-0,2}$

$\Lambda$

$P=200 \times 4=800$

La surestimation est très importante et le résultat trouvè est absurde. Notons que si l'efficacité en $2^{\text {e }}$ pêche est nettement supérieure à l'efficacité de la 1 ere pèche, la condition trouvée par LAURENT et LAMARQUE (1974) pour appliquer la méthode DeLURY n'est pas remplie; c'est le cas ici :

$\mathrm{C}_{1}=\mathrm{Px}=80 \quad \mathrm{C}_{2}=\mathrm{P}(1-\mathrm{x}) \mathrm{y}=72$

$\frac{C^{2}}{C_{1}^{2}} \frac{\left(C_{1}-C_{2}\right)^{2}}{\left(C_{1}+C_{2}\right)}=\frac{(80)^{2}}{(72)^{2}} \times \frac{(8)^{2}}{152}=0,5$.

Pour que la méthode s'applique, la quantité calculée doit être supérieure à 16. Dans le cas de l'exemple pris ici, la méthode n'est donc pas applicable.

\subsection{Méthode PETERSEN}

$m=P x$

$r=P x^{2}$

$u=P(1-x) y$ 


$$
P_{p}=\frac{m(u+r)}{r}=P . \frac{x^{2}+(1-x) y}{x} .
$$

Par le même raisonnement qu'au paragraphe 2.1 , on montre que :

$$
\begin{aligned}
& \Lambda \\
& P_{P}=P \times \frac{N}{D} \\
& N-D=(1-x)(y-x) .
\end{aligned}
$$

On aboutit aux mêmes conclusions

$y>x, N-D>0$, et $P p>P$ surestimation

$y<x, N-D<0$, et $P_{p}<P$ sous-estimation.

\section{Exemple numérique}

On reprend $P=200, x=$ efficacité 1 ere pêche et sur marqués $2^{e}$ pêche $=0,6 ; y=0,4$.

$$
\hat{P_{P}}=200 \times \frac{0,36+0,16}{0,6}
$$

$\Lambda$

$P_{P}=200 \times 0,87=174$.

$\Lambda$

L'estimation Pr sera 174, soit une sous-estimation de $13 \%$. on obtient :

Si avec la même population $P=200$, on suppose $x=0,4$ et $y=0,6$,

$$
\hat{P_{P}}=200 \times \frac{0,16+0,36}{0,4}=200 \times 1,3=260 .
$$

$\Lambda$

L'estimation Pr sera 260, soit une surestimation de $30 \%$.

\subsection{Conduite pratique du calcul}

Un exemple réel (population d'anguilles) permet de montrer le calcul de la correction à apporter à $P_{p}$, lorsque le nombre total de poissons capturés en deuxième pêche est inférieur à celui capturé en première pêche : dans ce cas, on est à peu près certainement dans l'hypothèse des calculs précédents. En effet, on peut admettre que les poissons déjà capturés en première pêche ont sensiblement les mêmes chances d'être capturés en deuxième pêche. La baisse du nombre total de poissons capturés en deuxième pêche est donc très certalnement imputable aux poissons non marqués.

$$
\mathrm{m}=\mathrm{Px} \quad \mathrm{r}=\mathrm{Px}^{2} \quad \mathrm{x}=\frac{\mathrm{r}}{\mathrm{m}}
$$




$$
\begin{aligned}
\text { d'où : } P=\frac{m^{2}}{r} \quad P_{p}=\frac{m(u+r)}{r} \\
\frac{P_{P}}{P}=\frac{u+r}{m} \\
\text { avec } m=89 \quad u=34 \quad r=48 \\
\frac{P p}{P}=\frac{82}{89}=0,92 \quad P_{P}=\frac{m(u+r)}{r}=152 .
\end{aligned}
$$

La valeur corrigée $P$ de $P$ p sera donc $P=\frac{P_{p}}{0,92}=\frac{152}{0,92}=165$.

On peut remarquer que $P$ aurait pu être calculé d'emblée :

$P=\frac{m^{2}}{r}=\frac{(89)^{2}}{48}=165$

L'efficacité sur les non marqués s'ècrit :

$$
y=\frac{\mathrm{u}}{\mathrm{P}-\mathrm{m}}=\frac{34}{165-89}=\frac{34}{76}=0.45
$$

On voit donc que dans la méthode PETERSEN, si la deuxième pêche donne un nombre total de poissons inférieur à la première, on doit calculer $P$ par la $\mathrm{m}^{2}$

formule $P=\frac{-}{r}$ (on peut noter que si $y=x$, conditions d'application de la méthode, $m=u+r$ et $\left.P_{r}=\frac{m(u+r)}{r}=P=\frac{m^{2}}{r}\right)$.

\subsection{Comparaison des deux estimations}

$\Lambda$

On formule le rapport $\frac{P_{L}}{\Lambda}$ :

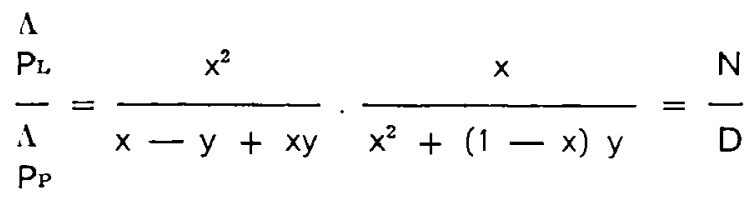

On forme $N-D$ :

$N-D=x^{3}-[x-(1-x) y]\left[x^{2}+(1-x) y\right]$,

tous calculs faits :

$N-D=y(1-x)^{2}(y-x)$ 
$N-D$ est du signe de $y-x$, donc :

$$
\begin{aligned}
y>x, N>D, \mathrm{PL}_{\mathrm{L}}>\mathrm{P}_{\mathrm{P}} \\
\mathrm{y}<\mathrm{x}, \mathrm{N}<\mathrm{D}, \mathrm{P}_{\mathrm{P}}>\mathrm{P}_{\mathrm{L}}
\end{aligned}
$$

Si on rapproche ces inégalités des résultats des $\S 2.1$ et 2.2 , on remarque $\begin{array}{ll}\text { que pour } y>x & P_{L}^{\prime}>P_{\mathrm{r}}>P\end{array}$

$$
\text { et que pour } y<x \quad P>\begin{aligned}
& 1 \\
& P_{1}>P_{L}
\end{aligned}
$$

Ceci montre que, en cas de sous-estimation, * PETERSEN " sous-estime moins que "DeLURY " et que, en cas de surestimation, "PETERSEN * surestime moins que * DeLURY. On peut donc en conclure que les erreurs dues aux variations de l'efficacité sont moins importantes dans le cas de a PETERSEN * que dans le cas de * DeLURY. Ceci est d'ailleurs bien visible sur l'exemple numérique :

a) $x=0,6 \quad y=0,4 \quad P=200$

$$
\begin{aligned}
& \Lambda \\
& P_{L}=164 \quad P_{P}=172 \text {. La sous-estimation * PETERSEN * est plus faible. } \\
& \text { b) } x=0,4 \quad y=0,6 \quad P=200
\end{aligned}
$$
$\Lambda$
$P_{L}=$ non déterminable $P_{P}=260$.
Dans ce cas, l'avantage de "PERTERSEN ॰ est évident.

\subsection{Conséquences pratiques}

$$
2.41 y>x
$$

Lorsquion se trouve devant un peuplement important les individus sont difficiles à capturer du fait de leur nombre. Dans le cas de la méthode "DeLURY ", en $2^{e}$ pêche, le nombre d'individus est plus restreint, ils sont plus facilement capturables et l'efficacité augmente. Dans la méthode a DeLURY . également, on a remarqué (CUINAT et al., 1975) que les opérateurs ont tendance à sélectionner les poissons sur leur taille, ainsi l'efficacité augmente sur les petites tailles, lors de la $2^{e}$ pêche. Enfin, et ceci est valable pour les deux méthodes et pour certaines espèces (l'anguille en particulier), il peut arriver qu'un poisson déjà choqué et non capturé en 1 ere pêche soit plus facilement capturable en ${ }^{\theta}$ pêche.

$$
2.42 y<x
$$

II s'agit du cas, envisagé classiquement (TIMMERMANS, 1957), où les poissons (en particulier les Salmonidés), déjà choqués et non capturés en ire pêche, sont d'autant moins capturables en $2 \mathrm{e}$ pêche. A ce propos, on peut observer qu'il n'y a pas de règle générale et que chaque espèce a un comportement propre devant le choc électrique. Dans ce cas, les deux estimations $\Lambda$ sous-estiment le peuplement, mais $\mathrm{PP}_{\mathrm{P}}$ (PETERSEN) sous-estime moins que $\mathrm{P}_{\mathrm{L}}$ (DeLURY). 


\section{EFFICACITES, AU COURS D'UNE MEME PECHE, DIFFERANT POUR DEUX SOUS-POPULATIONS}

Il s'agit du cas où une fraction de la population a une probabilité de capture différente du reste.

Soient $A$ et $B$ les 2 sous-populations :

$q$ : proportion de $A$ dans $P$

1 - $q$ : proportion de $B$ dans $P$

$X$ : efficacité pour $A$

$y$ : efficacité pour $B$,

$\overline{\mathrm{e}}=\mathrm{qx}+(1-q) \mathrm{y}=$ efficacité pondérée pour le peuplement en première pêche.

\subsection{Méthode DeLURY}

$C_{1}=q P x+(1-q) P y$

$C_{1}=P(q x+(1-q) y)=P e^{-}$

Après la première pêche la proportion de $A$ dans $P$ varie et devient $q^{\prime}$,

$$
\begin{aligned}
\text { avec } q^{\prime} & =\frac{P q-P q x}{P-P e} \\
q^{\prime} & =q \frac{1-x}{1-e^{-}}
\end{aligned}
$$

$C_{2}=q^{\prime}\left(P-C_{1}\right) x+\left(1-q^{\prime}\right)\left(P-C_{1}\right) y$

$C_{2}=P\left[(1-\bar{e}) q^{\prime} x+(1-\bar{e})\left(1-q^{\prime}\right) y\right]$

$C_{2}=P(1-\bar{e})\left(q^{\prime} x+\left(1-q^{\prime}\right) y\right)$.

soit $\bar{e}^{\prime}=q^{\prime} x+\left(1-q^{\prime}\right) y=$ efficacité pondérée en deuxième pêche.

$\mathrm{C}_{2}=\mathrm{Pe}^{-}(1-\overline{\mathrm{e}})$

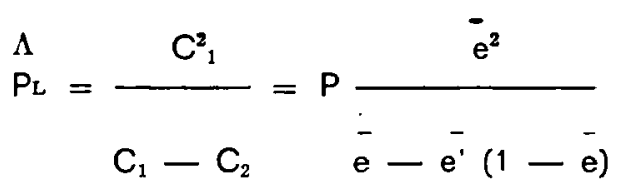

$\hat{P}_{\mathrm{L}}=\mathrm{P}$

$$
\bar{e}-\overline{e^{\prime}}+\bar{e} \bar{e}
$$


comme au $\S 2, \mathrm{P}_{\mathrm{L}}=\mathrm{P} \frac{\mathrm{N}}{\mathrm{D}}$

$$
\begin{aligned}
& N-D=\bar{e}^{2}-\bar{e}+\bar{e}-\bar{e} e^{\prime} \\
& =(1-\bar{e})(\bar{\theta}-\bar{e}) \\
& \overline{e^{\prime}}-\bar{e}=q^{\prime} x+\left(1-q^{\prime}\right) y-q x(1-q) y \\
& =x\left(q^{\prime}-q\right)+y\left(1-q^{\prime}-1+q\right)=(x-y)\left(q^{\prime}-q\right) \\
& q^{\prime}-q=q \frac{1-x}{1-\bar{e}}-q=q \frac{\bar{e}-x}{1-\bar{e}}=-q \frac{(x-y)(1-q)}{1-\bar{e}} .
\end{aligned}
$$

d'où :

$N-D=(1-e)(x-y)\left(q^{\prime}-q\right)=-q(1-q)(x-y)$.

$N-D$ est donc du signe du produit - q $(1-q)$ c'est-à-dire négatif, donc il y a toujours sous-estimation du peuplement.

\section{Exemple numérique}

Soit $P=200$ poissons, et une proportion $q=60 \%=0,6$ de poissons capturables avec une efficacité $x=0,6$ ou $60 \%$, les autres étant capturables avec une efficacité de $q=0,2$ ou $20 \%$.

$$
\begin{aligned}
& \bar{e}=q x+(1-q) y=0,6 \times 0,6+0,4 \times 0,2=0,44 \\
& q^{\prime}=q \frac{1-x}{1-e}=0,6 \times \frac{0,4}{0,56}=0,43 \\
& \overline{e^{\prime}}=0,43 \times 0,6+0,57 \times 0,2=0,37 \\
& \text { D'où } P_{L}=200 \times \frac{(0,44)^{2}}{0,44-0,37+0,44 \times 0,37}
\end{aligned}
$$

$\hat{\mathrm{P}_{\mathrm{L}}}=200 \times 0,84=168$.

La sous-estimation est de $16 \%$.

3.2 Méthode PETERSEN en supposant que les efficacités ne varlent pas entre les deux pêches.

$m=P e^{-}$ 


$$
\begin{aligned}
r= & P\left[q x^{2}+(1-q) y^{2}\right] \begin{array}{c}
\text { car la proportion ne varie pas entre les deux pêches } \\
\text { du fait de la remise à l'eau des poissons }
\end{array} \\
u= & P\left[\bar{e}-q x^{2}-(1-q) y^{2}\right] . \\
& \text { Si }: u+r=m
\end{aligned}
$$$$
\hat{P}_{p}=P \frac{\overline{e^{2}}}{q x^{2}+(1-q) y^{2}}
$$$$
\stackrel{A}{P p}=P \frac{N}{D}
$$$$
N-D=[q x+(1-q) y]^{2}-q x^{2}-(1-q) y^{2}
$$$$
=-q(1-q)(x-y)^{2} \text {. }
$$

$N$ - $D$ est toujours négatif, donc la méthode PETERSEN sous-estime toujours le peuplement et on observe que la quantité trouvée pour $N-D$ est identique à celle trouvée pour DeLURY.

\subsection{Comparaison des deux méthodes}

Du fait des considérations précédentes, on montre que, dans ce cas, l'estimation par la méthode PETERSEN donne le même résultat que celle obtenue par DeLURY : les deux quantités $N-D$ étant identiques et les $N$ également, il est évident que les deux $\frac{N}{D}$ le sont aussi.

\subsection{Evaluation de l'erreur}

$$
\begin{aligned}
& A=P\left(\frac{N}{D}\right) \\
& \Delta P=P_{P}-P=P\left(\frac{N-D}{D}\right) \\
& \frac{\Delta P}{P}=\text { erreur relative }=\frac{N-D}{D}=-\frac{q(1-q)(x-y)^{2}}{q\left(x^{2}-y^{2}\right)+y^{2}} .
\end{aligned}
$$

Soit E la fonction $\left|\frac{\Delta \mathrm{P}}{\mathrm{P}}\right|$

$$
E=\frac{q(1-q)(x-y)^{2}}{q\left(x^{2}-y^{2}\right)+y^{2}} \text {. }
$$


3.41 Si $y$ est petit devant $x$, on peut négliger le terme en $y^{2}$ au dénominateur.

$E \simeq \frac{q(1-q)(x-y)^{2}}{q\left(x^{2}-y^{2}\right)}=(1-q)\left(\frac{x-y}{x+y}\right)$

$E \simeq(1-q)\left(1-\frac{2 y}{x+y}\right)$

On obtient ainsi comme valeur maximum de l'erreur la quantité $1-q$. On retrouve ainsi un résultat prévisible : plus la proportion de la population la mieux capturable est grande, plus l'erreur d'estimation est faible. On peut prendre deux exemples :

a) $y=0,1 \quad x=0,6 \quad q=0,8$

(ce premier exemple correspond à un secteur dont la plus grande partie ( $80 \%$ ) est facile à pêcher $(x=0,6)$, le reste ètant constitué de zones profondes ou peu accessibles).

$E=0,2 \times\left(1-\frac{0,2}{0,7}\right)=0,14$.

L'erreur sur le peuplement estimé est donc $14 \%$, qu'il faut ajouter.

b) $y=0,1 \quad x=0,6 \quad q=0,5$.

Il s'agit d'un secteur constitué pour moitié de zones facilement et difficilement pêchables.

$E=0,5 \times\left(1-\frac{0,2}{0,7}\right)=0,35$.

L'erreur est donc ici de $35 \%$; le peuplement est beaucoup plus sousestimé que dans le cas précédent.

\subsection{Si $x$ est petit devant $y$,}

$E \simeq \frac{q(1-q)(x-y)^{2}}{(1-q) y^{2}}$

$E \simeq q\left(1-\frac{x}{y}\right)^{2}$

On obtient ici comme valeur maximum de l'erreur la quantité q. En d'autres termes, plus l'efficacité sur la population la plus représentée est faible, plus l'erreur est importante, ce qui est un résultat logique. On peut prendre deux exemples comme précédemment :

a) $y=0,6 \quad x=0,1 \quad q=0,8$

$E \simeq 0,8\left(1-\frac{0,1}{0,6}\right)^{2}=0,55$ ou $55 \%$. 
II s'agit ici du cas où le secteur de rivière étudié comprendrait surtout les zones difficiles à pêcher.

b) $y=0,6 \times=0,1 \quad q=0,5$

$E \simeq 0,5 \times\left(1-\frac{0,1}{0,6}\right)^{2}=0,34$ ou $34 \%$.

Notons ici que ce cas est le même que celui du paragraphe 3.41.b. La légère différence trouvée provient des approximations. De toutes façons, lorsqu'on considère que l'une des deux efficacités n'est pas faible devant l'autre, il faut pour calculer $E$ utiliser la formule générale donnée au paragraphe 3.4.

\subsection{Conséquences pratiques}

Le cas étudié ici se présente très fréquemment dans les opérations pratiques d'un inventaire piscicole. Les différences d'efficacité peuvent provenir de la taille des poissons : l'erreur peut alors être éliminée en calculant des efficacités différentes pour chaque groupe de taille (CUINAT et al., 1975). Elles peuvent aussi provenir du biotope : zones d'un même secteur où les facilités de captures sont différentes. II faut alors évaluer la proportion des zones difficiles dans le secteur ainsi que les deux efficacités.

\section{CONCLUSION}

Les erreurs d'estimation proviennent d'une manière générale d'un non respect des hypothèses d'application de lois statistiques utilisées pour calculer ces estimations. Dans le cas de l'inventaire piscicole, les variations d'efficacité sont les causes d'erreurs les plus fréquentes. Les remarques données ici n'ont pas pour but de remédier systématiquement à ces inconvénients, mais d'attirer l'attention des utilisateurs sur les limites des possibilités d'erreurs dans l'emploi des estimations PETERSEN et DeLURY. Ces considérations peuvent néanmoins être utiles pour corriger les populations les plus probables estimées d'après les méthodes classiques.

\section{REFERENCES BIBLIOGRAPHIQUES}

CUINAT R., J. DUMAS, J.A. TIMMERMANS, J. ARRIGNON et G. TUFFERY, 1975. Diagnose écologique en cours d'eau à salmonidés. Méthode et exemple. Doc. tech. CECPI, 22, $122 \mathrm{p}$.

LAURENT $M$. et P. LAMARQUE, 1974. Utilisation de la méthode des captures successives (DeLURY) pour l'évaluation des peuplements piscicoles. Ann. Hydrobiol., 5, 2 : 121-132; et Bull. fr. Piscic., 1975, 259 : 66-77.

TIMMERMANS J.A., 1957. Estimation des populations piscicoles. Application aux eaux courantes rhéophiles. Trav. Stn. rech. Groenendaal, D., 21, 84 p. 